\title{
Influence of spin-orbit coupling on the magnetic dipole term $T_{\alpha}$
}

\author{
O. Šipr, ${ }^{1,}{ }^{*}$ J. Minár, ${ }^{2,3}$ and H. Ebert ${ }^{2}$ \\ ${ }^{1}$ Institute of Physics ASCR v.v.i., Cukrovarnická 10, CZ-162 53 Prague, Czech Republic \\ ${ }^{2}$ Department Chemie, Universität München, Butenandtstrasse 5-13, D-81377 München, Germany \\ ${ }^{3}$ New Technologies Research Centre, University of West Bohemia, Pilsen, Czech Republic
}

(Received 25 July 2016; published 10 October 2016)

\begin{abstract}
The influence of the spin-orbit coupling (SOC) on the magnetic dipole term $T_{\alpha}$ is studied across a range of systems in order to check whether the $T_{\alpha}$ term can be eliminated from analysis of x-ray magnetic circular dichroism spectra performed via the spin moment sum rule. Fully relativistic Korringa-Kohn-Rostoker Green's function calculations for Co monolayers and adatoms on $\mathrm{Cu}, \mathrm{Pd}, \mathrm{Ag}, \mathrm{Pt}$, and $\mathrm{Au}$ (111) surfaces were performed to verify whether the sum over magnetic dipole terms $T_{x}+T_{y}+T_{z}$ is zero and whether the angular dependence of the $T_{\alpha}$ term goes as $3 \cos ^{2} \theta-1$. It follows that there are circumstances when the influence of the SOC on $T_{\alpha}$ cannot be neglected even for $3 d$ atoms where the SOC is nominally small. The crucial factor appears to be the dimensionality of the system: For $3 d$ adatoms, the influence of SOC on $T_{\alpha}$ can be significant whereas for monolayers it is always practically negligible. Apart from the dimensionality, hybridization between adatom and substrate states is also important: Small hybridization enhances the importance of the SOC and vice versa.
\end{abstract}

DOI: 10.1103/PhysRevB.94.144406

\section{INTRODUCTION}

Magnetism of diluted and low-dimensional systems, such as adatoms, clusters, or monolayers, is one of the strongly pursued research areas. Magnetization of these systems often cannot be measured by macroscopic methods. It can, however, be probed indirectly by making use of spectroscopy. One of the most powerful methods in this respect is X-ray magnetic circular dichroism (XMCD). It consists of measuring the difference in the absorption of left- and right-circularly polarized $\mathrm{x}$ rays in a magnetized sample while the energy of the incident $\mathrm{X}$ rays is varied. Analysis of XMCD spectra is often performed with the help of sum rules, which link integrals of XMCD and $\mathrm{x}$-ray absorption spectral peaks to local spin and orbital magnetic moments. Most of the recent progress in magnetism of atomic-sized systems is associated with the application of the XMCD sum rules [1-3].

The strength of the sum rules is that they provide, in the case of $L_{2,3}$ edge spectra, separate information about the orbital magnetic moment $\mu_{\text {orb }}$ and the spin magnetic moment $\mu_{\text {spin }}$ of the photoabsorbing atom [4,5]. However, extracting values of $\mu_{\text {orb }}$ and, especially, of $\mu_{\text {spin }}$ from the spectra is not straightforward. Considering the most common case of the $L_{2,3}$ edge spectra and a sample magnetized along the $\alpha$ direction $(\alpha=x, y, z)$, the spin magnetic moment sum rule can be written as [5]

$$
\frac{3}{I} \int\left(\Delta \mu_{L_{3}}-2 \Delta \mu_{L_{2}}\right) d E=\frac{\mu_{\mathrm{spin}}+7 T_{\alpha}}{n_{h}},
$$

where $\Delta \mu_{L_{2,3}}$ are the differences $\Delta \mu=\mu^{(+)}-\mu^{(-)}$between absorption coefficients for the left- and right-circularly polarized light propagating along the $\alpha$ direction, $I$ is the integrated isotropic absorption spectrum, $\mu_{\text {spin }}$ is the local spin magnetic moment (its $d$ component, to be precise), and $n_{h}$ is the number of holes in the $d$ band. The term $T_{\alpha}$ is the expectation value of the intra-atomic spin dipole operator for the valence

\footnotetext{
*sipr@fzu.cz; http://www.fzu.cz/ sipr
}

$d$ electrons. It is often called the magnetic dipole term in the literature dealing with XMCD. As the magnetization is typically in the $\alpha=z$ direction, one often speaks simply about the $T_{z}$ term.

This magnetic dipole $T_{\alpha}$ term can be written as [6,7]

$$
\begin{aligned}
T_{\alpha} & =-\frac{\mu_{B}}{\hbar}\left\langle\hat{T}_{\alpha}\right\rangle \\
& =-\frac{\mu_{B}}{\hbar}\left\langle\sum_{\beta} Q_{\alpha \beta} S_{\beta}\right\rangle,
\end{aligned}
$$

with

$$
Q_{\alpha \beta}=\delta_{\alpha \beta}-3 r_{\alpha}^{0} r_{\beta}^{0}
$$

being the quadrupole moment operator and $S_{\alpha}$ being the spin operator. The $T_{\alpha}$ term cannot be easily determined by experiment, and its occurrence in Eq. (1) thus poses a serious problem. For bulk systems, it can be often neglected (provided that the spin-orbit coupling (SOC) is not very strong [8]). However, for low-dimensional systems it can be significant [9-11]. Moreover, the $T_{\alpha}$ term cannot be considered just as an additive correction that for similar systems simply shifts the values of $\mu_{\text {spin }}$ by approximately the same amount. It was demonstrated that neglecting $T_{\alpha}$ for a sequence of supported magnetic clusters could lead to erroneous conclusions regarding the dependence of the average $\mu_{\text {spin }}$ on the cluster size [12]. Likewise, neglecting $T_{\alpha}$ and its angular dependence could introduce spurious anisotropy of $\mu_{\text {spin }}$ for low-dimensional systems $[6,13,14]$.

In principle, the $T_{\alpha}$ term can be calculated and inserted into Eq. (1). However, one would really have to make the calculation for each system which is studied because the $T_{\alpha}$ term is quite sensitive to details of the electronic structure [9,10,12] and taking its values from calculations for only similar systems might not be reliable. At the same time, performing calculations for exactly the system one is interested in may be difficult or impractical.

Fortunately, there appears to be a way to eliminate the $T_{\alpha}$ term from Eq. (1) relying solely on experiment by performing 
a series of angle-dependent XMCD measurements. The key here lies in decoupling the quadrupole moment operator $\hat{Q}$ in Eq. (2) from the spin operator $\hat{S}$. This can be performed provided that the influence of the SOC on $T_{\alpha}$ can be neglected. Then, for a sample magnetically saturated along the direction $\alpha$, one can express the $T_{\alpha}$ term as [15]

$$
T_{\alpha}=\sum_{m} \frac{1}{2}\left\langle Y_{2 m}\left|\hat{Q}_{\alpha \alpha}\right| Y_{2 m}\right\rangle \mu_{\mathrm{spin}}^{(m)},
$$

where $\mu_{\text {spin }}^{(m)}$ is the spin magnetic moment resolved into components according to the magnetic quantum number $m$. The matrix elements $\left\langle Y_{2 m}\left|\hat{Q}_{\alpha \alpha}\right| Y_{2 m}\right\rangle$ can be found in Stöhr and König [15], and a more elaborate discussion of Eq. (4) can be found in Stöhr [7] or Šipr et al. [14]. Elimination of the $T_{\alpha}$ term from the sum rule (1) can then be achieved by performing three XMCD measurements and making use of the relation [15],

$$
T_{x}+T_{y}+T_{z}=0 .
$$

Furthermore, if the system has higher than twofold symmetry around the $z$ axis, the magnetic dipole term depends on the polar angle $\theta$ as $[15,16]$

$$
T_{\theta} \approx 3 \cos ^{2} \theta-1 \text {. }
$$

The magnetic dipole term, which we will denote $T_{\theta}$ for a general direction in which the sample is magnetically saturated, can thus be eliminated by a single XMCD measurement with circularly polarized $x$ rays coming in the direction of the magic angle $54.7^{\circ}$. This approach was employed, e.g., for studying Co thin films and nanoclusters $[13,17]$.

The important point is that eliminating $T_{\alpha}$ from the sum rule analysis is possible only if the effect of SOC on $T_{\alpha}$ can be neglected. The question is whether this happens in common circumstances. Namely, there are theoretical indications that the effect of SOC on $T_{\alpha}$ may be sometimes important. It was found that Eq. (5) is strongly violated for free-standing Co wires [18] (provided that correlation effects beyond the localdensity approximation are included via the Brooks orbital polarization term [19]). For more realistic materials, violation of Eq. (6) was predicted for a Pt monolayer with magnetization induced from an Fe substrate [20]. Not surprisingly, this violation is even more serious for systems with very strong SOC, such as US [8,20]. Recently, there have been also experimental indications that the SOC may be important for the $T_{\alpha}$ term: Violation of Eq. (5) was observed for the low-temperature monoclinic phase of magnetite nanoparticles [21].

The most typical situation when XMCD sum rules are used is studying magnetism of $3 d$ metals in multicomponent systems, and the $T_{\alpha}$ term has to be considered especially for thin films, adatoms, or clusters. One should thus explore to what extent the SOC is important for $T_{\alpha}$ in these systems so that one knows whether Eqs. (5) and (6) can be applied to eliminate the $T_{\alpha}$ term from the XMCD analysis or not.

To get a comprehensive view, we focus on a sequence of systems comprising $\mathrm{Co}$ monolayers and $\mathrm{Co}$ adatoms on $\mathrm{Cu}$, $\mathrm{Pd}, \mathrm{Ag}, \mathrm{Pt}$, and $\mathrm{Au}$ (111) surfaces. In that way we account for effects connected with the change in dimensionality and for effects connected with the changes in chemical environment as well. It should be noted in this context that the substrate may have a crucial influence on some SOC-induced properties, such as the magnetocrystalline anisotropy [22]. There is also theoretical evidence that the substrate has a decisive influence on $T_{\alpha}$ of the supported systems [12].

The outline of the paper is the following. We start by describing our computational framework. Then we present results that are in line with Eqs. (5) and (6) for a series of Co monolayers and adatoms. Here we demonstrate that whereas for Co monolayers the effect of SOC on $T_{\alpha}$ can be neglected for any of the investigated substrates, the situation is complicated for Co adatoms where for some substrates Eqs. (5) and (6) are valid whereas for others they are not. This outcome is reinforced by inspection of the validity of the approximate relation (4) for $T_{\alpha}$. Finally, we investigate the density of states (DOS) to get an understanding of why for adatoms on some substrates Eqs. (5) and (6) are valid whereas for adatoms on other substrates they are not.

\section{COMPUTATIONAL SCHEME}

The calculations were performed within the ab initio spindensity functional theory framework, relying on the local spindensity approximation (LSDA) with the Vosko-Wilk-Nusair parametrization for the exchange and correlation potential [23]. The electronic structure is described, including all relativistic effects, by the Dirac equation, which is solved using the spin-polarized relativistic multiple-scattering or KorringaKohn-Rostoker (KKR) Green's-function formalism [24] as implemented in the SPR-TB-KKR code [25]. The potentials were treated within the atomic sphere approximation (ASA), and for the multipole expansion of the Green's function, an angular momentum cutoff of $\ell_{\max }=3$ was used. The energy integrals were evaluated by contour integration on a semicircular path within the complex energy plane using a logarithmic mesh of 32 points. The integration over the $\mathbf{k}$ points was performed on a regular mesh using 10000 points in the full surface Brillouin zone.

We deal with systems which do not have three-dimensional periodicity, therefore, special approaches have to involved. Calculations for supported monolayers are accomplished by means of the tight-binding or screened KKR method [26]. The substrate was modeled by a finite slab of 16 atomic layers, the vacuum was represented by 4 layers of empty sites (this corresponds to $9 \AA$ ). Calculations for adatoms were accomplished by means of embedded impurity formalism: First the electronic structure of the host system (clean surface) was calculated, and then a Dyson equation for an embedded impurity cluster was solved [27]. The impurity cluster contains 131 sites; this includes one Co atom, 70 substrate atoms, and the rest are empty sites. This cluster defines the zone in which the electrons are allowed to react to the presence of the adatom; there is an unperturbed host beyond this zone.

We investigate a series of Co adatoms and Co monolayers on the (111) surface of the noble metals $\mathrm{Cu}, \mathrm{Ag}, \mathrm{Au}, \mathrm{Pd}$, and Pt. In this way we include in our study substrates which are hard to magnetically polarize $(\mathrm{Cu}, \mathrm{Ag}, \mathrm{Au})$, substrates that are easy to polarize $(\mathrm{Pd}, \mathrm{Pt})$, as well as substrates with weak SOC $(\mathrm{Cu})$, with moderate SOC $(\mathrm{Pd}, \mathrm{Ag})$, and with strong $\mathrm{SOC}(\mathrm{Pt}, \mathrm{Au})$. We assume that all atoms are located 
on ideal lattice sites of the underlying bulk fcc lattice; no structural optimization was attempted. Although this would affect comparison of our data with experiment, we do not expect this to have a significant influence on the conclusions. Likewise, relying on the ASA does not significantly restrict the validity of our analysis because our focus is on the SOC and the effects of SOC are most pronounced in the region close to atomic nuclei where the potential is approximately spherically symmetric.

\section{RESULTS}

\section{A. Sum over magnetic dipole term components $T_{\alpha}$}

The first test of the influence of SOC on the $T_{\alpha}$ term is checking the validity of Eq. (5). Our motivation comes from the spin moment sum rule Eq. (1) in which $\mu_{\text {spin }}$ appears only in combination with $7 T_{\alpha}$ as $\mu_{\text {spin }}+7 T_{\alpha}$. The key indicator is thus the ratio $7 T_{\alpha} / \mu_{\text {spin. }}$. Table I shows this ratio summed over all three coordinates $\sum_{\alpha=x, y, z} 7 T_{\alpha} / \mu_{\text {spin }}$. It should be zero if the influence of SOC on $T_{\alpha}$ can be neglected.

One can see that for Co monolayers the condition (5) is fulfilled with a high accuracy. However, the situation changes for Co adatoms. It is obvious from Table I that the ratio $\sum_{\alpha} 7 T_{\alpha} / \mu_{\text {spin }}$ is significantly larger for adatoms than for the corresponding monolayers. For Pd and Pt substrates the breakdown of Eq. (5) is modest. However, for $\mathrm{Cu}, \mathrm{Ag}$, and $\mathrm{Au}$ substrates this breakdown is substantial. The physical reason for this difference is discussed in Sec. III D 2 below.

The breakdown of Eq. (5) for adatoms is not related to any specific choice of the coordinate system. Similar numbers as those shown in Table I are obtained if the sum over three coordinate axes is substituted by a corresponding integral over the full space angle (cf. also Fig. 1 below). It should be also noted that the dependence of the spin moment alone on the magnetization direction is negligible: The variations do not exceed $0.03 \%$ for monolayers and $0.4 \%$ for adatoms.

\section{B. Angular dependence of the magnetic dipole term}

Another view on the same problem can be obtained by inspecting the angular dependence of the magnetic dipole term $T_{\theta}$. Figure 1 shows the $T_{\theta}$ term calculated while varying the angle $\theta$ between the magnetization direction and the surface normal. The azimuthal angle $\phi$ was kept at $0^{\circ}$ with the $x$ axis parallel to the [101] direction. If the influence of SOC can be neglected, the $T_{\theta}$ dependence should satisfy Eq. (6). Therefore, we tried to fit our $a b$ initio data to the expression,

$$
A\left(3 \cos ^{2} \theta-1\right)
$$

TABLE I. Sum $7\left(T_{x}+T_{y}+T_{z}\right)$ devided by $\mu_{\text {spin }}$ for Co monolayers and Co adatoms on noble-metal surfaces.

\begin{tabular}{lcc}
\hline \hline Substrate & Monolayer & Adatom \\
\hline $\mathrm{Cu}$ & 0.011 & 0.206 \\
$\mathrm{Pd}$ & 0.015 & 0.072 \\
$\mathrm{Ag}$ & 0.021 & 0.372 \\
$\mathrm{Pt}$ & 0.008 & 0.098 \\
$\mathrm{Au}$ & 0.009 & 0.284 \\
\hline \hline
\end{tabular}
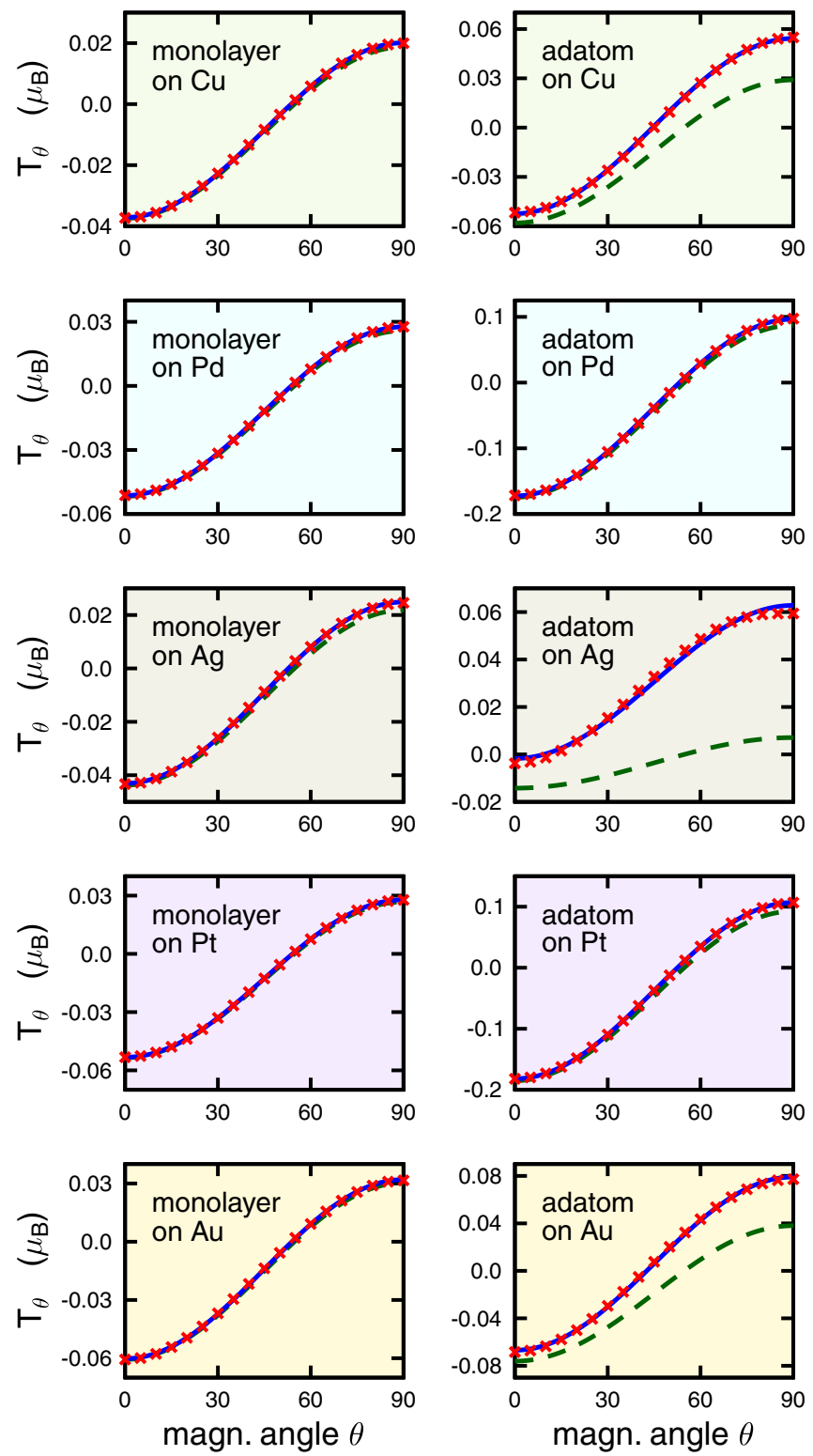

FIG. 1. Dependence of the magnetic dipole term $T_{\theta}$ on the magnetization angle $\theta$ for Co monolayers (left panels) and Co adatoms (right panels) on different substrates. $A b$ initio results are shown by red marks, fits to $A\left(3 \cos ^{2} \theta-1+B\right)$ are shown by full blue lines, and fits to $A\left(3 \cos ^{2} \theta-1\right)$ are shown by dashed green lines. Both fits are practically undistinguishable except for the cases of adatoms on $\mathrm{Cu}, \mathrm{Ag}$, or $\mathrm{Au}$.

(dashed green lines in Fig. 1). This fit is quite accurate except for $\mathrm{Co}$ adatoms on $\mathrm{Cu}, \mathrm{Ag}$, and $\mathrm{Au}$. In these cases the $T_{\theta}$ dependence can be fitted with the function,

$$
A\left(3 \cos ^{2} \theta-1+B\right)
$$

(full blue lines in Fig. 1).

The fact that the $T_{\theta}$ dependence can be fitted by Eq. (6) only if a rigid shift (represented by the constant $B$ ) is introduced presents another evidence that the magnetic dipole term sum rule (5) is not universally valid for supported $3 d$ systems.

Likewise, for systems where the parameter $B$ in Eq. (8) is important, the term $T_{\theta}$ does not vanish at the "universal magic 
TABLE II. Magnetic dipole term for $\boldsymbol{M} \| x\left(T_{x}\right)$ and $\boldsymbol{M} \| z\left(T_{z}\right)$ evaluated using the exact expression (2) and using the approximate relation (4).

\begin{tabular}{|c|c|c|c|c|c|}
\hline \multirow[b]{2}{*}{ Substrate } & & \multicolumn{2}{|c|}{ Co monolayer } & \multicolumn{2}{|c|}{ Co adatom } \\
\hline & & Exact & Approximate & Exact & Approximate \\
\hline \multirow[t]{2}{*}{$\mathrm{Cu}$} & $T_{x}$ & 0.020 & 0.021 & 0.057 & 0.031 \\
\hline & $T_{z}$ & -0.037 & -0.042 & -0.052 & -0.061 \\
\hline \multirow[t]{2}{*}{$\mathrm{Pd}$} & $T_{x}$ & 0.028 & 0.027 & 0.099 & 0.093 \\
\hline & $T_{z}$ & -0.051 & -0.055 & -0.173 & -0.187 \\
\hline \multirow[t]{2}{*}{$\mathrm{Ag}$} & $T_{x}$ & 0.025 & 0.024 & 0.059 & 0.008 \\
\hline & $T_{z}$ & -0.043 & -0.048 & -0.004 & -0.016 \\
\hline \multirow[t]{2}{*}{$\mathrm{Pt}$} & $T_{x}$ & 0.028 & 0.028 & 0.109 & 0.098 \\
\hline & $T_{z}$ & -0.053 & -0.055 & -0.184 & -0.196 \\
\hline \multirow[t]{2}{*}{$\mathrm{Au}$} & $T_{x}$ & 0.032 & 0.032 & 0.080 & 0.040 \\
\hline & $T_{z}$ & -0.061 & -0.064 & -0.066 & -0.079 \\
\hline
\end{tabular}

angle" $54.7^{\circ}$. Rather, the magnetization tilt angle for which $T_{\theta}$ vanishes differs from substrate to substrate: It is $45^{\circ}$ for a $\mathrm{Co}$ adatom on $\mathrm{Cu}, 13^{\circ}$ for an adatom on $\mathrm{Ag}$, and $42^{\circ}$ for an adatom on $\mathrm{Au}$. For other systems explored here, it is close to $54.7^{\circ}$.

\section{Approximate relation for $T_{\alpha}$ in terms of $\mu_{\text {spin }}^{(m)}$}

Getting an intuitive insight into the $T_{\alpha}$ term by relying on the exact Eq. (2) is not easy. The approximate Eq. (4) is far better suited for this purpose. It presents $T_{\alpha}$ as a linear combination of orbitally projected components of the spin magnetic moment $\mu_{\text {spin }}^{(m)}$, illustrating thus the frequently used interpretation of the magnetic dipole term as the manifestation of the anisotropy of spin-density distribution. Indeed, if all $m$ components of $\mu_{\text {spin }}$ are identical, $T_{\alpha}$ is zero.

However, this view is transparent only if the effect of SOC on $T_{\alpha}$ can be neglected. Therefore we present in Table II a comparison between values of $T_{\alpha}$ obtained by evaluating the exact Eq. (2) and by evaluating the approximate Eq. (4). We focus on two magnetization directions $\boldsymbol{M} \| x$ and $\boldsymbol{M} \| z$. For $\boldsymbol{M} \| y$, the results are practically the same as for $\boldsymbol{M} \| x$. One can see from Table II that as concerns Co monolayers, the approximate equation yields similar values as the exact equation. For Co adatoms, the agreement is worse and, again, it depends on the substrate. For adatoms on $\mathrm{Pd}$ and $\mathrm{Pt}$, the validity of the approximate equation is worse than for corresponding monolayers, but it is still acceptable. However, for adatoms on $\mathrm{Cu}, \mathrm{Ag}$, and $\mathrm{Au}$ the error of the approximate Eq. (4) reaches $50 \%-100 \%$.

Comparison of the exact and approximate values of $T_{x}$ and $T_{z}$ in Table II can serve as another indicator of the role of SOC for the magnetic dipole term. The outcome of this analysis is consistent with the conclusions based on inspection of Eq. (5) in Sec. III A and Eq. (6) in Sec. III B. Namely, the influence of the SOC on the $T_{\alpha}$ term can be neglected for monolayers on any substrate and for adatoms on Pd and Pt, whereas it has to be taken into account when dealing with $T_{\alpha}$ for adatoms on $\mathrm{Cu}, \mathrm{Ag}$, and $\mathrm{Au}$.

\section{Density of states}

\section{Total spin-polarized DOS}

To summarize, we found two trends concerning the impact of SOC on $T_{\alpha}$. First, the dimensionality or perhaps better the size of the system is crucial: The effect of SOC can be always neglected for monolayers but only sometimes for adatoms. Second, there is a big variance depending on the substrate, but the nominal strength of the substrate SOC does not seem to be important.

Thinking about the explanation, one should realize that in metals the SOC strength $\xi$ should be compared to the bandwidth. In our case that means to the bandwidths for $3 d$ electrons $W_{3 d}$; it is the $\xi / W_{3 d}$ ratio that matters $[15,28]$. The bandwidth $W_{3 d}$ depends on the hybridization, i.e., on how electronic states around the $3 d$ atom are affected by its neighbors. An idea how this varies across our systems can be obtained by inspecting the DOS. Therefore we present in Fig. 2 the spin-polarized DOS for all the systems we investigate. Apart from the DOS for Co atoms we show also the DOS for the nearest substrate atoms so that hybridization between them can be studied.

One can see that (not surprisingly) the bandwidth for monolayers is always significantly larger than the bandwidth for adatoms, no matter what is the substrate. This clarifies why the influence of SOC on the $T_{\alpha}$ term is negligible for the monolayers: In that case, the effect of the hybridization always overwhelms the effect of SOC.

\section{DOS overlap integrals}

What is not clear is why there are so big differences for the adatoms when going from one substrate to another. The bandwidth associated with adatoms as shown in Fig. 2 is approximately the same for all substrates. One can, nevertheless, quantify the importance of hybridization between the adatom and the substrate by evaluating the DOS overlap integral, i.e., the integral of the product of the DOS for the adatom $n_{\mathrm{Co}}^{(s)}$ and for the nearest substrate atom $n_{\text {subs }}^{(s)}$,

$$
h^{(s)} \equiv \int d E n_{\mathrm{Co}}^{(s)}(E) n_{\text {subs }}^{(s)}(E),
$$

where $s$ stands for the spin. Higher $h^{(s)}$ means higher hybridization and, consequently, larger effective bandwidth-even though it may not be apparent visually in Fig. 2. An elucidating relation between the integrals $h^{(s)}$ and the importance of the SOC for $T_{\alpha}$ can be identified if we focus on the minority-spin states $(s=\downarrow)$. In particular, the relative importance of SOC for $T_{\alpha}$ (quantified as the ratio $\sum_{\alpha} 7 T_{\alpha} / \mu_{\text {spin }}$, cf. Sec. III A and Table I) is approximately proportional to the degree of atomiclike character of minority-spin adatom states (which can be quantified as $\left.1 / h^{(\downarrow)}\right)$. This is demonstrated in Table III where relative weights of $\sum_{\alpha} 7 T_{\alpha} / \mu_{\text {spin }}$ and $1 / h^{(\downarrow)}$ are shown. One can thus see that if adatom states are less hybridized with the substrate leading to smaller effective bandwidth, the relative importance of SOC increases - in agreement with intuition.

The only caveat here is that this correspondence holds only for minority-spin states; if majority-spin states are included in the analysis, the correspondence between $\sum_{\alpha} 7 T_{\alpha} / \mu_{\text {spin }}$ and 

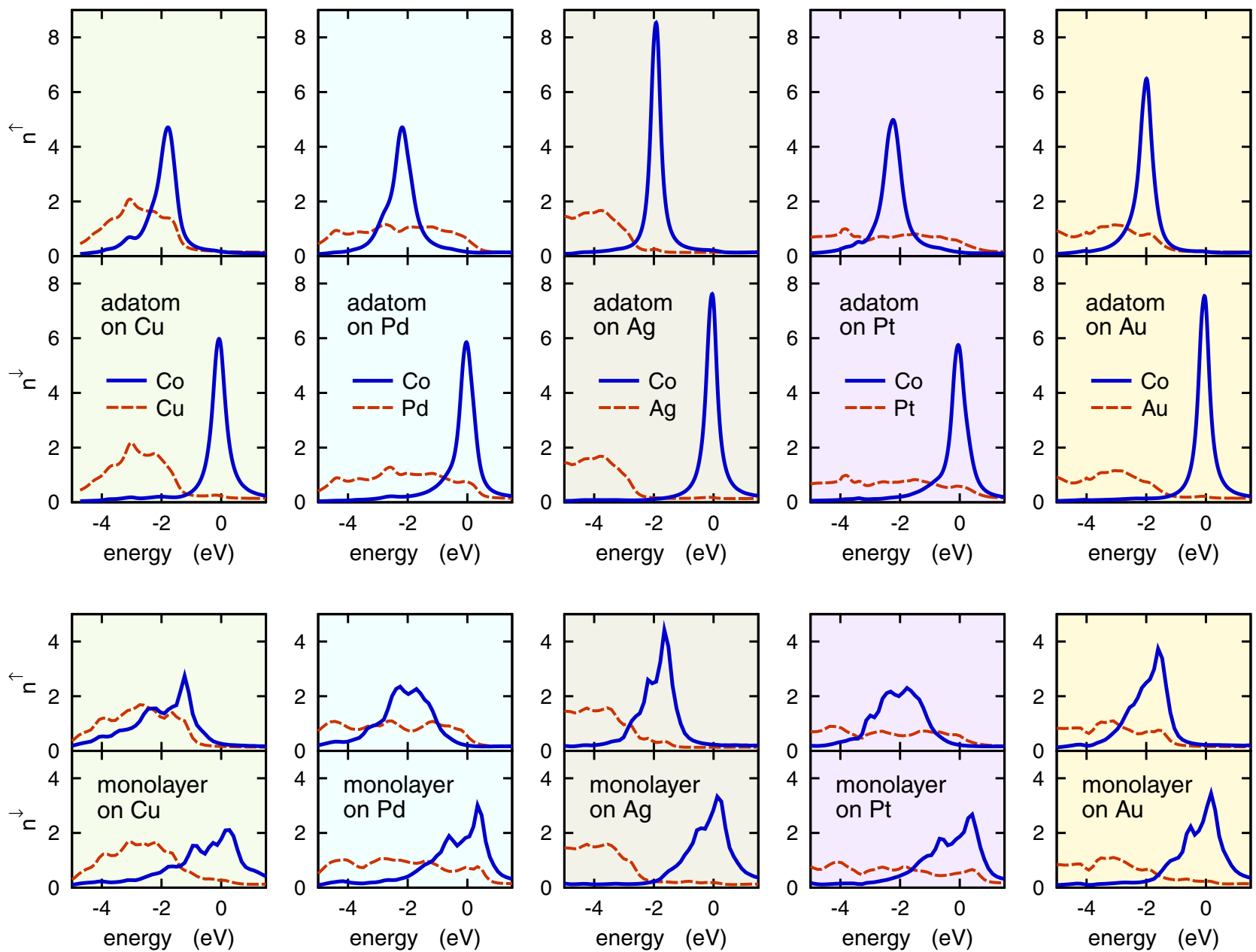

FIG. 2. Spin-polarized DOS for Co adatoms (upper panels) and monolayers (lower panels) on noble metals. The blue solid lines show the DOS for Co atoms (in states per eV), and the dashed brown lines show the DOS for those substrate atoms which are nearest neighbors to Co atoms.

$1 / h^{(s)}$ disappears. However, there is a reason for focusing on minority-spin states only. If majority-spin states are mostly occupied (as it is the case for our systems), it is the incomplete occupancy of minority-spin states which induces asphericity. The importance of partially filled minority-spin states for $T_{\alpha}$ is also emphasized by the fact that the value of $T_{\alpha}$ strongly depends on the position of $E_{F}$, which cuts through minorityspin states $[9,10,12]$.

TABLE III. Comparing the importance of SOC for $T_{\alpha}$ (characterized by sums over three $T_{\alpha}$ components, the second column) to the degree of atomiclike character of states associated with the adatom (characterized by reciprocal values of the DOS overlap integrals, the third column).

\begin{tabular}{lcc}
\hline \hline & $\begin{array}{c}\text { Relative weight of } \\
\sum_{\alpha} 7 T_{\alpha} / \mu_{\text {spin }}\end{array}$ & $\begin{array}{c}\text { Relative weight of } \\
1 / \int d E n_{\text {Co }}^{\downarrow}(E) n_{\text {subs }}^{\downarrow}(E)\end{array}$ \\
\hline $\mathrm{Cu}$ & 0.181 & 0.197 \\
$\mathrm{Pd}$ & 0.061 & 0.091 \\
$\mathrm{Ag}$ & 0.390 & 0.324 \\
$\mathrm{Pt}$ & 0.092 & 0.117 \\
$\mathrm{Au}$ & 0.276 & 0.269 \\
\hline \hline
\end{tabular}

\section{Orbitally resolved DOS for Co adatoms}

Yet another view on hybridization of adatom states with substrate states can be obtained from the orbitally resolved DOS for the Co adatom. This is shown in Fig. 3: Majority-spin states are inspected in the upper panels, and minority-spin states are inspected in the lower panels. Several features in this plot are worth commenting on. First, the individual orbital-resolved peaks are broader for the majority-spin states than for these minority-spin states. This is because for the majority-spin states there is a considerable overlap with substrate states whereas for the minority-spin states there is practically no overlap (see Fig. 2). The Ag substrate with a deep lying $d$ band is an exception, and in this case the majorityspin adatom states have no overlap with substrate states either.

The DOS peaks for $m= \pm 2$ resemble broadened energy levels as for an isolated atom. This is because the orbital lobes for $m= \pm 2$ lie parallel to the surface where there are no other atoms to hybridize with. The situation for $m=0$ is similar-here the orbital lobe points to the void between three nearest substrate atoms. The influence of the substrate is most pronounced for the $m= \pm 1$ orbitals, whose lobes are directed toward neighboring atoms. Apart from that, states for $m= \pm 1$ 

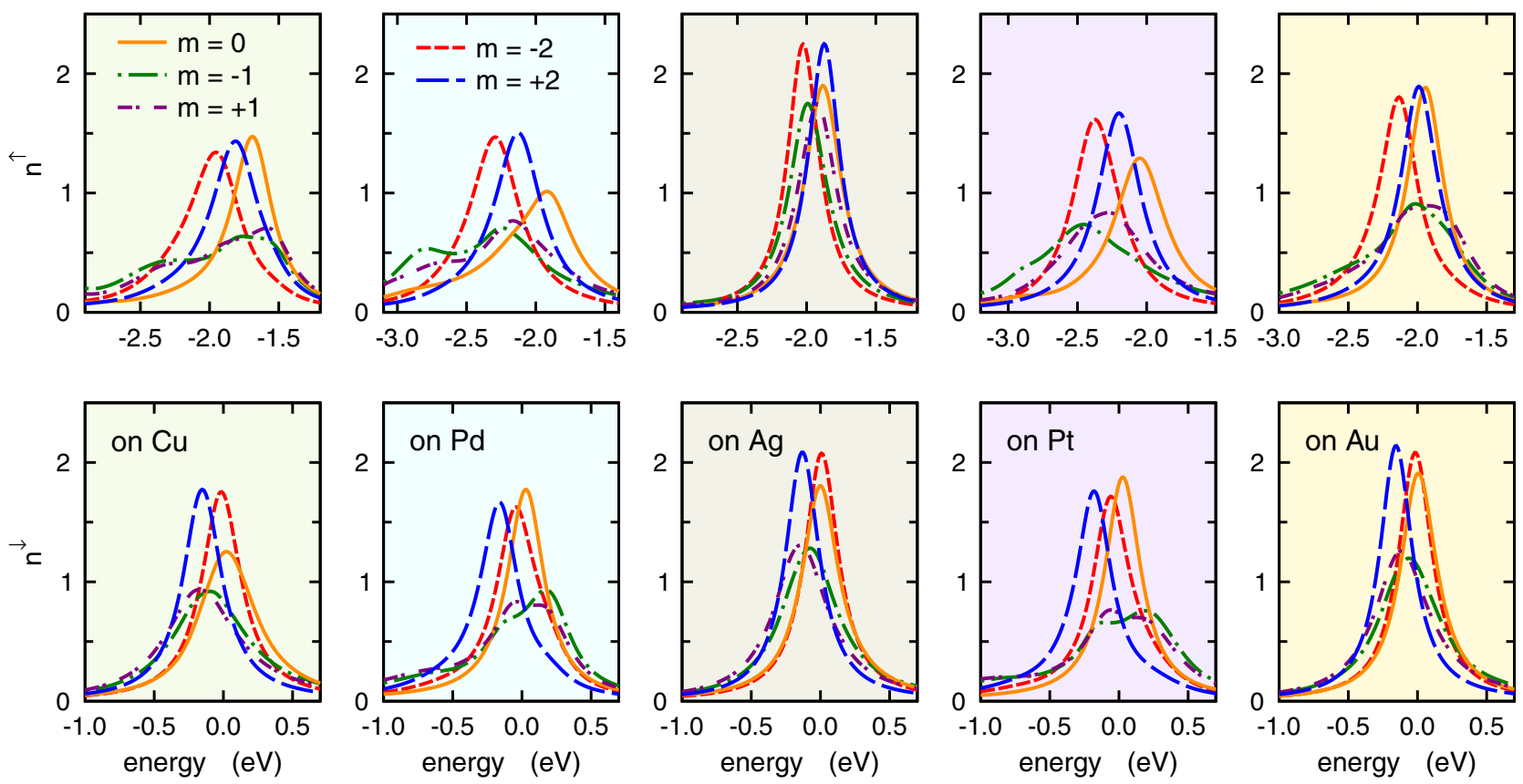

FIG. 3. Orbitally resolved DOS (in states per eV) for Co adatoms on noble-metal surfaces. Majority-spin $d$ states are shown in the upper panels, and minority-spin $d$ states are shown in the lower panels. Meaning of the lines is shown in the two upper left panels.

and $m= \pm 2$ are split by the SOC. A more formal discussion about resolving the DOS according to spin and orbital quantum numbers as well as about the role of the SOC-induced splitting for the magnetocrystalline anisotropy was recently presented by Šipr et al. [28].

Here our attention is on the hybridization and, in particular, on the difference among $\mathrm{Cu}, \mathrm{Ag}$, and Au substrates on one hand and Pd and Pt substrates on the other hand. This difference is apparent for the minority-spin states with $m= \pm 1$ (lower panels of Fig. 3): Whereas there is only one single peak for each of the $m= \pm 1$ components for the $\mathrm{Cu}, \mathrm{Ag}$, and $\mathrm{Au}$ substrates, and there are two peaks for the Pd and Pt substrates (each of them about $0.15 \mathrm{eV}$ either below and above $E_{F}$ ). We can infer from this that the hybridization is very small in the case of a Co adatom on $\mathrm{Cu}, \mathrm{Ag}$, and $\mathrm{Au}$ whereas it is relatively large in the case of a Co adatom on Pd and Pt. Consequently, the SOC has a large role for the $T_{\alpha}$ term for adatoms on $\mathrm{Cu}, \mathrm{Ag}$, and $\mathrm{Au}$ and only a small role for the $T_{\alpha}$ term for adatoms on Pd and Pt. An analysis of the orbital-resolved DOS thus reinforces the message obtained by analyzing the overlap integrals (9) in Table III.

\section{DISCUSSION}

The validity of the XMCD sum rules and specifically the role of the $T_{\alpha}$ term were the subject of several earlier studies $[9,12,29]$. The purpose of this paper was to focus on the $T_{\alpha}$ term itself and to study systematically the conditions under which the influence of SOC on it can be neglected for low-dimensional $3 d$ systems. Fulfillment of this condition is, namely, necessary for eliminating the $T_{\alpha}$ term from the spin moment sum rule (1) altogether. We found that even for atoms with low SOC, such as Co, the influence of SOC on $T_{\alpha}$ in certain environments can be so large that Eqs. (5) and (6) cannot be used. The crucial factor turns out to be the ratio between the SOC and the bandwidth $\xi / W_{3 d}$. This subsequently translates itself into the dependence on the dimensionality. It turns out that for Co monolayers the influence of SOC on $T_{\alpha}$ can be neglected for any of the $\mathrm{Cu}, \mathrm{Pd}, \mathrm{Ag}, \mathrm{Pt}$, or $\mathrm{Au}$ substrates. We assume that this is true for any $3 d$ monolayer on any substrate. Similar conclusions were drawn earlier for bulk $3 d$ systems and surfaces [20].

For adatoms the situation is more complicated. The narrowing of the $3 d$ band caused by the decrease in the dimensionality appears to be just of that amount which is required for SOC to become important for $T_{\alpha}$. Hence details of the electronic structure of the substrate begin to matter; for some substrates (Pd,Pt) Eqs. (5) and (6) still can be used whereas for others $(\mathrm{Cu}, \mathrm{Ag}, \mathrm{Au})$ they cannot. The hybridization between adatom and substrate states around $E_{F}$ seems to be the deciding factor. We expect that for systems with considerable overlap between adatom and substrate DOS around $E_{F}$ (minority-spin states in our case, see Fig. 2) the influence of SOC on $T_{\alpha}$ can be neglected even for adatoms. Otherwise Eqs. (5) and (6) should rather not be used.

To find more about when the size of the system gets so small that Eqs. (5) and (6) cannot be used anymore, we performed calculations also for a $\mathrm{Co}$ wire on $\mathrm{Au}(111)$. The wire was built along the $[1 \overline{1} 0]$ direction, and we modeled it by a $2 \times 1$ surface supercell. To test whether Eq. (5) could be applied for such a system, we evaluated the ratio $\sum_{\alpha} 7 T_{\alpha} / \mu_{\text {spin }}$ and found it to be 0.058 [30]. This is to be compared with 0.284 for a monolayer and 0.009 for an adatom (see Table I). We conclude, therefore, that the borderline between systems which satisfy Eqs. (5) and (6) and which do not is somewhere between the wire and the adatom. When analyzing XMCD spectra for small $3 d$ clusters of just a few atoms, one should not rely on Eqs. (5) and (6). When analyzing XMCD spectra of clusters of hundreds of atoms (as was the case, e.g., in the study of Koide et al. [17]), reliance on Eqs. (5) and (6) is justified. 


\section{CONCLUSIONS}

The influence of spin-orbit coupling on the magnetic dipole term $T_{\alpha}$ can be neglected for $3 d$ transition-metal systems as long as they are sufficiently large. If the system contains just a few $3 d$ atoms (as is the case of adatoms or small supported clusters), the influence of SOC on $T_{\alpha}$ may be significant. This further depends on the hybridization between states of the $3 d$ atoms and of the substrate, especially around the Fermi level: If the hybridization is only weak, the role of the SOC is enhanced whereas if the hybridization is strong, the role of the SOC is suppressed. For systems where the influence of SOC on $T_{\alpha}$ cannot be neglected, the $T_{\alpha}$ term cannot be eliminated from the XMCD spin sum rule-neither by relying on the $T_{x}+T_{y}+T_{z}=0$ relation, nor by making use of the magic angle $\theta=54.7^{\circ}$.

\section{ACKNOWLEDGMENTS}

This work was supported by the Ministry of Education, Youth and Sport (Czech Republic) within the Projects No. LD15097 (O.Š.) and No. LO1402 (J.M.) and by the Deutsche Forschungsgemeinschaft within the Project SFB 689 "Spinphänomene in reduzierten Dimensionen."
[1] P. Gambardella, S. Rusponi, M. Veronese, S. S. Dhesi, C. Grazioli, A. Dallmeyer, I. Cabria, R. Zeller, P. H. Dederichs, K. Kern, C. Carbone, and H. Brune, Science 300, 1130 (2003).

[2] F. Donati, A. Singha, S. Stepanow, C. Wäckerlin, J. Dreiser, P. Gambardella, S. Rusponi, and H. Brune, Phys. Rev. Lett. 113, 237201 (2014).

[3] F. Donati, S. Rusponi, S. Stepanow, C. Wäckerlin, A. Singha, L. Persichetti, R. Baltic, K. Diller, F. Patthey, E. Fernandes, J. Dreiser, Ž. Šljivančanin, K. Kummer, C. Nistor, P. Gambardella, and H. Brune, Science 352, 318 (2016).

[4] B. T. Thole, P. Carra, F. Sette, and G. van der Laan, Phys. Rev. Lett. 68, 1943 (1992).

[5] P. Carra, B. T. Thole, M. Altarelli, and X. Wang, Phys. Rev. Lett. 70, 694 (1993).

[6] J. Stöhr, J. Electron. Spectrosc. Relat. Phenom. 75, 253 (1995).

[7] J. Stöhr, J. Magn. Magn. Materials 200, 470 (1999).

[8] S. P. Collins, D. Laundy, C. C. Tang, and G. van der Laan, J. Phys.: Condens. Matter 7, 9325 (1995).

[9] R. Wu and A. J. Freeman, Phys. Rev. Lett. 73, 1994 (1994).

[10] M. Komelj, C. Ederer, J. W. Davenport, and M. Fähnle, Phys. Rev. B 66, 140407(R) (2002).

[11] S. Stepanow, A. Mugarza, G. Ceballos, P. Moras, J. C. Cezar, C. Carbone, and P. Gambardella, Phys. Rev. B 82, 014405 (2010).

[12] O. Šipr, J. Minár, and H. Ebert, Europhys. Lett. 87, 67007 (2009).

[13] D. Weller, J. Stöhr, R. Nakajima, A. Carl, M. G. Samant, C. Chappert, R. Mégy, P. Beauvillain, P. Veillet, and G. A. Held, Phys. Rev. Lett. 75, 3752 (1995).

[14] O. Šipr, S. Bornemann, H. Ebert, S. Mankovsky, J. Vackář, and J. Minár, Phys. Rev. B 88, 064411 (2013).

[15] J. Stöhr and H. König, Phys. Rev. Lett. 75, 3748 (1995).
[16] G. van der Laan, Phys. Rev. B 57, 5250 (1998).

[17] T. Koide, H. Miyauchi, J. Okamoto, T. Shidara, A. Fujimori, H. Fukutani, K. Amemiya, H. Takeshita, S. Yuasa, T. Katayama, and Y. Suzuki, Phys. Rev. Lett. 87, 257201 (2001).

[18] C. Ederer, M. Komelj, J. W. Davenport, and M. Fähnle, J. Electron. Spectrosc. Relat. Phenom. 130, 97 (2003).

[19] M. S. S. Brooks, Physica B 130, 6 (1985).

[20] T. Oguchi and T. Shishidou, Phys. Rev. B 70, 024412 (2004).

[21] D. Schmitz, C. Schmitz-Antoniak, A. Warland, M. Darbandi, S. Haldar, S. Bhandary, O. Eriksson, B. Sanyal, and H. Wende, Sci. Rep. 4, 5760 (2014).

[22] O. Šipr, S. Bornemann, H. Ebert, and J. Minár, J. Phys.: Condens. Matter 26, 196002 (2014).

[23] S. H. Vosko, L. Wilk, and M. Nusair, Can. J. Phys. 58, 1200 (1980).

[24] H. Ebert, D. Ködderitzsch, and J. Minár, Rep. Prog. Phys. 74, 096501 (2011).

[25] H. Ebert and R. Zeller, The SPR-TB-KKR package, http://olymp.cup.uni-muenchen.de (2006)

[26] R. Zeller, P. H. Dederichs, B. Újfalussy, L. Szunyogh, and P. Weinberger, Phys. Rev. B 52, 8807 (1995).

[27] S. Bornemann, J. Minár, S. Polesya, S. Mankovsky, H. Ebert, and O. Šipr, Phase Transitions 78, 701 (2005).

[28] O. Šipr, S. Mankovsky, S. Polesya, S. Bornemann, J. Minár, and H. Ebert, Phys. Rev. B 93, 174409 (2016).

[29] C. Piamonteze, P. Miedema, and F. M. F. de Groot, Phys. Rev. B 80, 184410 (2009).

[30] Our finding that SOC is not very important for $T_{\alpha}$ for a Co wire on $\mathrm{Au}(111)$ is not in contradiction with the results of Ederer et al. [18] because they studied a free-standing Co wire and employed the Brooks orbital polarization correction. 